\title{
Subject Index to Volume 31
}

Acid-base balance, effect of varying arterial oxygen tension, neonatal swine, 112

Acidification, developmental changes, juxtamedullary proximal convoluted tubule, rabbit, 411

Acylcarnitine, medium chain, urinary analysis, medium chain acyl-CoA dehydrogenase deficiency, 545

Acyl-coenzyme A dehydrogenase deficiency medium chain

diagnosis, radioisotopic exchange/ HPLC method, 545

gas chromatographic diagnosis, lymphocytes and liver, 39

immunoblot analysis of fibroblast MCAD from, 34

mutation, 305

short chain, tissue-specific isoforms, $\mathrm{J}$ mutant mice, 552

Adolescents, insulin-dependent diabetic, evaluation, dawn phenomenon, 203

Adrenal gland, steroidogenesis, metalloporphyrin effect, rat, 196

$\beta$-Adrenergic receptor, myocardial responsiveness, thyroid hormone effect, fetal lamb, 468

Airway

smooth muscle, maturational changes, sheep, 151

structure-function, preterm lamb, 157

Alcohol, acute maternal intoxication, cerebral response, fetal sheep, 645

Antioxidant enzyme, hyperoxic esposure effect, alveolar and whole lung cells, neonatal and adult rat, 441

Aorta, coarctation repair, reversibility of hypertensive vascular changes after, dog, 297

Apnea, reflex, respiratory syncytial virus effect, young lamb, 381

Arginine vasopressin, effect on ion transport fetal distal lung epithelium, rat, 318

Asphyxia, cerebral changes after, fetal sheep, 486

Atrial natriuretic peptide, effect on ion transport, fetal distal lung epithelium, rat, 318

Auditory brainstem response, outcome predictor, very low birth weight infants, 68

Basic fibroblast growth factor, role, fetal lung development, rat, 435

Biotinidase, serum levels, characterization, partial biotinidase deficiency, 261

Blood coagulation: see Coagulation

Blood flow

cerebral

pancuronium bromide effect during seizures, newborn pig, 636

velocity assessment, fetus and neonate, 649

choroidal, indomethacin effect, during hypercarbia, newborn piglet, 127

distribution, extracorporeal membrane oxygenation effect, neonatal lamb, 80

pulmonary, response to infusion of exogenous adenosine and ATP, fetal lamb, 451 retinal, indomethacin effect, during hypercarbia, newborn piglet, 127

uteroplacental, increased red cell aggregation effect, pregnant guinea pig, 91

Blood group $\mathrm{A}$, antigenic determinants, in surfactant protein-A, 364

Blood monocyte, expression of surfactant tissue factor apoprotein by, during infection, early infancy, 567

Bone

formation, serum bone $\gamma$-carboxyglutamic acid protein as marker of, breast-fed $v s$ formula-fed infants, 401

mineralization, outcome, human milk-fed preterm infants, 583

Bone marrow, transplantation, severe Gaucher disease, 503

Bone mineral content, prediction, very low birth weight infants, 181

Brain: see also specific anatomical location

fetal glucose transport, intrauterine growth retardation, rat, 59

regional alterations, galactose-1-phosphate uridyltransferase, rat, 512

regulation of mammalian glucose transporters, 1

Brain damage, hypoxic-ischemic, hypoglycemia effect, newborn rat, 138

Bronchopulmonary dysplasia, marker, type III collagen, $\mathrm{N}$-terminal propeptide of, 340

Calcium, inhibiting effect on iron absorption, human and cow's milk, 524

Capsule, role, group B Streptococcus-induced pulmonary hypertension, 386

Carbon dioxide, transcutaneous, premature and normal term infants, 73

$\gamma$-Carboxyglutamic acid protein, serum bone concentrations, breast-fed vs formulafed infants, 401

Cardiac cycle, length, ventricular relaxation rate and, chick embryo, 480

Cardiomegaly, dexamethasone-induced, neonatal rat, 359

Carnitine, role, prophylaxis of valproate hepatotoxicity, mouse, 419

Carotid artery, denervation, febrile response altered by, lamb, 107

Catecholamine, plasma concentration, capillary $v s$ arterial, as sympatho-adrenal marker, infant, 579

Catheter, conductance volume, left ventricular volume measurement, piglet, 85

Cerebral cortex, pial arteriolar dilation, after topical application of group B streptococci, rat, 640

Cholestasis, chronic, enhancement of vitamin D absorption. 146

Cholesterol, plasma levels, term neonate, 396

Chronic granulomatous disease, $\mathrm{X}$-linked recessive, genetic heterogeneity in patients with, 516

Coagulation

activation, possible mechanism, sepsis, infant, 567

impairment of thrombin regulation, sick neonates, 391
Coarctation, repair, reversibility of hypertensive vascular changes after, dog, 297

Collagen, type III, N-terminal propeptide, tracheal fluid, preterm infants, 340

Colony-stimulating factor, granulocyte-macrophage, decreased production, human neonatal $T$ cells, 211

Colostrum, human, histidine-rich glycoprotein in, 239

Complex carbohydrates, abnormal galactosylation of, fibroblasts from galactosemia patients, 508

Copper, inherited toxicity, animal model of Wilson's disease, rat, 253

CSF: see Colony-stimulating factor

Cystic fibrosis, erythrocyte sodium-potassium transport, 425

Cytokine, -induced pulmonary hypertension pentoxifylline effect, piglet, 163

Cytopenia, pathogenesis, during sepsis, 574

Dawn phenomenon, comparison, normal and insulin-dependent diabetic adolescents, 203

Deoxyribonucleic acid

confirmation, original dried blood specimens, neonatal hemoglobinopathy screening, 217

mitochondrial deletions, Kearns-Sayre syndrome and Pearson's marrowpancreas syndrome, 557

Dexamethasone, cardiomegaly induced by, neonatal rat, 359

Diabetes

insulin-dependent

dawn phenomenon, adolescents, 203

maternal, effect on neonatal glycerol metabolism and triglyceride-fatty acid cycling, 52

Diaphragm, glycogen content, increased work load effect, neonatal lamb, 354

Duodenum, immunoglobulin A response, SIDS victims, 372

Eicosanoid, -induced pulmonary hypertension, pentoxifylline effect, piglet, 163

Elastin, pulmonary vascular, developmental study, rat, 280

Energy expenditure, malaria and fever effect, Gambian children, 102

Enteral feeding, preterm infant, intestinal motor activity, fasting and feeding, 587

Enterotoxin, jejunal secretory response, malnourished rat, 228

Enzyme

augmentation, Gaucher disease, 496

urea cycle, human fetal liver, gestational age and, 143

Epidermal barrier, maturation, lipid content and bilayer organization and, perinatal rat, 308

Epidermal growth factor, RNA ontogeny and, thyroxine treatment effect, rat kidney, 330

Epidermal growth factor receptor, RNA ontogeny and, thyroxine treatment effect, rat kidney, 330 
Epinephrine, intratracheal, age-related pulmonary changes, rabbit, 458

Epithelia, noncutaneous, separation, junctional epidermolysis bullosa, fetus, 561

Erythrocyte

increased aggregation impact, placental blood flow, pregnant guinea pig, 91

sodium-potassium transport, cystic fibrosis, 425

Erythropoiesis, peripheral blood, growth characteristics, 170

Escherichia coli, heat-stable enterotoxin, jejunal secretory response, malnourished rat, 228

Ethanol, teratogenesis, mechanism of, 492

Extracorporeal membrane oxygenation, effect on blood flow distribution, neonatal lamb, 80

Fasting, hepatic protein synthesis and, suckling rat, 247

Fat, regulation of mammalian glucose transporters, 1

Fatty acid ethyl ester, role, fetal alcohol syndrome, 492

Fetal alcohol syndrome, fatty acid ethyl ester role, 492

Fetus

asphyxia, cerebral changes after, sheep, 486

cerebral flow velocity waveforms, perinatal assessment, 649

cerebral response, acute maternal alcohol intoxication, sheep, 645

circulating placental lactogen, nutritional regulation, 520

growth-retarded

cerebral and adrenal monoamine metabolism, rat, 131

glucose transport, rat, 59

hypothyroidism, postnatal cardiovascular responsiveness and, lamb, 468

insulin exposure, tissue iron status and, rat, 64

junctional epidermolysis bullosa, noncutaneous epithelia with, 561

late-gestation lung, transforming growth factor- $\alpha$ gene expression, rat, 286

liver, urea cycle enzyme levels, gestational age and, 143

lung

blood flow, ATP and adenosine effect, lamb, 45 I

development, platelet-derived growth factor role, rat, 323

expression of basic fibroblast growth factor, rat, 435

reflectance pulse oximetry, lamb, 266

$\mathrm{T}_{3}$ sulfate as source of $\mathrm{T}_{3}$, rat tissue, 54

Fever

carotid denervation effect, young lamb, 107

effect on energy expenditure, malaria patients, Gambia, 102

Fumarylacetoacetase, immunoreactive protein, hereditary tyrosinemia type I, 43

Galactosemia, abnormal galactosylation in, 508

Gas chromatography, diagnosis of medium chain acyl-CoA dehydrogenase deficiency, lymphocytes and liver, 39

Gastric mucosa, thickness, age-related changes, rat, 193

Gaucher disease

allogenic bone marrow transplantation, 503

enzyme augmentation, 496

GBS: see Streptococcus, group B
G-CSF: see Granulocyte-colony stimulating factor

Glucose, mammalian transporters, regulation of, 1

Glycerol, neonatal metabolism, effect of maternal diabetes and intrauterine growth retardation, 52

Glycogen, intramuscular use, increased work load effect, neonatal diaphragm, lamb, 354

Glycoprotein, histidine-rich, identification, human colostrum and milk, 239

Gonadotropin, nocturnal profiles, prediction, prepubertal and early pubertal boys, 535

Gonadotropin-releasing hormone, low dose, response, prepubertal and early pubertal boys, 535

Granulocyte-colony stimulating factor, production, mononuclear cells, adult and neonate, 574

Growth and development

airway smooth muscle, maturational changes, sheep, 151

auditory brainstem response and outcome, very low birth weight infants, 68

cardiac relaxation, developmental regulation, rabbit, 474

cartilage-hair hypoplasia, evaluation and growth charts for, 176

cerebral flow velocity wave forms, fetus and neonate, 649

cerebral response to acute maternal alcohol intoxication, immature and mature fetal sheep, 645

epidermal barrier, critical events associated with, perinatal rat, 308

fetal lung

basic fibroblast growth factor role, rat, 435

platelet-derived growth factor role, rat, 323

gastric mucus gel thickness, developmental changes, rat, 193

G-CSF and IL-3 production, mononuclear cells, adult and neonate, 574

growth hormone receptor, tissue-specific developmental regulation, rat kidney and lung, 335

hepatic protein synthesis, response to fasting, suckling rat, 247

hyperoxic exposure effect, alveolar cells, neonatal and adult rat, 441

hypoxemia effect, fetal and adult hemoglobin synthesis, sheep, 483

juxtamedullary proximal convoluted tubule, acidification changes, rabbit, 411

lung fibroblast, prostaglandin and cAMP accumulation, rat, 344

maternal protein-calorie malnutrition effect, fetal and neonatal rat lung, 629

myocardial cholinergic signaling, age-related changes, sheep, 601

peripheral blood erythropoiesis, growth characteristics, 170

phosphodiesterase inhibitors in rabbit myocardium, newborn, immature, and adult, 300

preterm intestine, during fasting and feeding, 587

prolactin effect on $\mathrm{Na}^{+} \mathrm{K}^{+}$-A TPase activity, rat nephron, 207

prolonged growth hormone administration effect, chronically uremic rat, 406

pulmonary changes, after intratracheal epinephrine, rabbit, 458

receptor regulation, newborn sheep, 462

tropoelastin gene expression, rat pulmonary vasculature, 280 unsaturated triacylglycerol accumulation, early postnatal growth, rat, 47

urea cycle enzyme levels, gestational age and, fetal liver, 143

Growth hormone

deficiency, prevalence of hGH-l gene deletions in patients with, 532

gene deletion, prevalence, different populations, 532

prolonged administration, rats with chronic renal insufficiency, 406

secretion, effects of chronic renal insufficiency, rat, 528

tissue responsiveness, differential regulation, rat kidney and lung, 335

Growth hormone binding protein, developmental regulation, tissue-specific regulation, rat kidney and lung, 335

Growth hormone receptor, developmental regulation, tissue-specific regulation, rat kidney and lung, 335

Growth retardation

intrauterine

cerebral and adrenal monoamine metabolism effect, rat fetus, 131

effect on neonatal glycerol metabolism and triglyceride-fatty acid cycling, 52

fetal glucose transport, rat lung and brain, 59

Heart

left ventricular volume, measurement, conductance volume catheter, piglet, 85

sarcoplasmic reticulum calcium pump, developmental regulation, rabbit, 474

ventricular relaxation rate, cardiac cycle length and, chick embryo, 480

Heart rate

dynamic analysis, normal infants versus SIDS victims, 606

fluctuations, stroke volume and, preterm infants, 117

variability, congenital central hypoventilation syndrome, 291

Hemoglobin, synthesis, hypoxemia effect, late gestation lamb, 483

Hemoglobinopathy, screening program, DNA confirmation, dried blood specimens, neonate, 217

Hepatic protein: see Protein

Hepatitis, spontaneous, inherited copper toxicity, rat, 253

Hepatocyte, regulation of mammalian glucose transporters, 1

Herpes simplex virus, antibodies, comparison, human milk and serum, 591

Heterogeneity, genetic, patients with Xlinked recessive chronic granulomatous disease, 516

Hormone: see also Growth hormone

gastrointestinal, plasma concentrations, term and preterm infants, 587

Human immunodeficiency virus, binding to CD4 receptor, inhibition by human milk factor, 22

Hyperammonemia, ibuprofen interaction with, animal model of Reye's syndrome, ferret, 258

Hypercarbia, choroidal and retinal blood flow during, indomethacin effect, newborn piglet, 127

Hypercholesterolemia, plasma lathosterol levels and, preterm infants given Intralipid, 186

Hyperoxia, antioxidant enzyme activities and, alveolar cells, neonatal and adult rat, 441 
Hypertension

pulmonary

GBS-induced, capsule role, 386

GBS-induced, leukotriene $\mathrm{D}_{4}$ role, newborn lamb, 121

pentoxifylline effect, piglet, 163

Hypoglycemia, perinatal hypoxic-ischemic brain damage and, newborn rat, 138

Hypothyroidism, fetal, postnatal cardiovascular responsiveness and, lamb, 468

Hypoventilation, congenital central syndrome, heart rate variability, 29

Hypoxemia

chronic, postnatal intestinal function during, newborn lamb, 234

fetal hemoglobin synthesis and, late gestation lamb, 483

Hypoxia, neonatal acid-base balance and, swine, 112

Ibuprofen, interaction with influenza and hyperammonemia, animal model, Reye's syndrome, ferret, 258

IL-3: see Interleukin-3

Immune system, natural killer cell activity, very low birth weight infants, 376

Immunoglobulin $A$, response in duodenal mucosa, SIDS victims, 372

Immunoglobulin $M$, response in tracheal wall, SIDS victims, 372

Indomethacin

effect on pulmonary bacterial deposition, piglet, 14

effect on retinal and choroidal blood flow, during hypercarbia, newborn piglet, 127

-pentoxifylline pretreatment, GBS sepsis, piglet, 222

Infant: see also Neonate

preterm

heart rate fluctuations, 117

human milk-fed, bone mineralization outcomes, 583

hypercholesterolemia and plasma lathosterol, 186

intestinal motor activity, during fasting and feeding, 587

N-terminal propeptide of type III collagen measurement, tracheal fluid, 340 respiratory training effect, 613

whole-body protein parameters, comparison, tracer substances, 95

sympatho-adrenomedullary activity, assessment, capillary plasma catecholamines, 579

ventilatory regulation, normal changes, premature and normal term infants, 73

very low birth weight

auditory brainstem response failure, 68

natural killer cell activity, 376

prediction, developing bone mineral content, 181

Infant feeding, serum bone $\gamma$-carboxyglutamic acid protein concentrations, breast-fed $v s$ formula-fed infants, 401

Infection, expression of surface factor apoprotein and, blood monocytes, early infancy, 567

Influenza, ibuprofen interaction with, animal model of Reye's syndrome, ferret, 258

Inositol, role, pulmonary abnormalities, malnourished neonatal rat, 629

Insulin

rance role dawn phenomenon normal and insulin-dependent diabetic adolescents, 203

in utero exposure, tissue iron status and, rat, 64
Interleukin-3

decreased production, mononuclear cells, neonate, 574

production, mononuclear cells, adult and neonate, 574

Interleukin-6, defective production, neonatal monocyte, 18

Iron

bioavailability, human and cow's milk, role of calcium content, 524

status, effect of in utero insulin exposure, fetal rat tissue, 64

Isoproterenol, cAMP accumulation and, rat perinatal lung fibroblast, 344

Junctional epidermolysis bullosa, noncutaneous epithelia in, fetus, 561

Kearns-Sayre syndrome, blood mitochondrial DNA deletions, 557

Ketogenesis, from lactate, perinatal rat liver, 415

Kidney

chronic insufficiency

effect on growth hormone secretion, rat, 528

prolonged growth hormone administration effect, rat, 406

epidermal growth factor levels, RNA ontogeny, thyroxine effect, rat, 330

regulation of mammalian glucose transporters, 1

Lactate, ketogenesis from, perinatal rat liver, 415

Lathostero

plasma levels

hypercholesterolemia indicator, preterm infants given Intralipid, 186

term neonate, 396

Leukotriene $\mathrm{D}_{4}$, role, GBS-induced pulmonary hypertension, newborn lamb, 121

Lipid

hydrophobic, cutaneous barrier maturation and perinatal rat, 308

supplementation, pulmonary abnormalities, malnourished neonatal rat, 629

Liver

bactericidal activity, prostaglandin $H$ synthase effect, piglet, 14

chronic cholestatic disease, enhancement of vitamin D absorption, 146

hepatic protein synthesis, developmental changes and fasting effect, suckling rat, 247

medium-chain acyl-CoA dehydrogenase deficiency, gas chromatographic diag nosis, 39

role, lactate metabolism, perinatal rat, 415

toxicity, pantothenate and carnitine prophylaxis, mouse, 419

unsaturated triacylglycerol accumulation, early postnatal rat, 47

urea cycle enzyme levels, gestational age and, fetus, 143

Lung

age-related changes, after intratracheal epinephrine, rabbit, 458

bactericidal activity, prostaglandin $\mathrm{H}$ synthase effect, piglet, 14

blood flow, A TP and adenosine effect, fetal lamb, 451

compliance, distribution of surfactant and preterm rabbit, 270

development, platelet-derived growth factor role, rat, 323

distal epithelium, arginine vasopressin effect, fetal rat culture, 318 fetal development, basic fibroblast growth factor role, rat, 435

fetal glucose transport, intrauterine growth retardation, rat, 59

fibroblast, cAMP accumulation, prostaglandin and isoproterenol effect, rat, 344

growth and maturation, maternal proteincalorie malnutrition effect, rat, 629

mechanics, time dependence of, preterm lamb, 276

regulation of mammalian glucose transporters, 1

transforming growth factor- $\alpha$ gene expression, late-gestation fetal rat, 286

vascular properties, pulsatile perfusion effect, newborn lamb, 349

vasculature, tropoelastin gene expression, rat, 280

vasodilation, purine nucleotide effect, fetal lamb, 451

Lymphocyte, medium-chain acyl-CoA dehydrogenase deficiency, gas chromatographic diagnosis, 39

Malaria, effect on energy expenditure, Gambian children, 102

Malnutrition

protein-calorie, maternal, effect on fetal and neonatal rat lung, 629

role, jejunal secretory response to $E$. coli enterotoxin, 228

Maturation: see Growth and development

Meconium aspiration syndrome, surfactant lavage, piglet model, 625

Metalloporphyrins, effect on steroidogenesis comparison, tin and zinc, rat adrenal 196

Milk

human

anti-herpes simplex specific antibodies, 591

bone mineralization outcomes in pre term infants receiving, 583

histidine-rich glycoprotein in, 239

inhibition of HIV binding to CD4 receptor by, 22

tumor necrosis factor- $\alpha$ in, 29

Monoamine, cerebral and adrenal metabolism, growth-retarded rat fetus, 131

Monocyte, neonatal, defective production of interleukin- 6 by, 18

Mononuclear cells, granulocyte-CSF and IL3 expression from, comparison, adult neonate, 574

Motor activity, during fasting and feeding, intestine, preterm infant, 587

Muscie, regulation of mammalian glucose transporters,

Myocardium

cholinergic-linked signaling response, agedependent change, sheep, 601

phosphodiesterase inhibitors, intropic responses, newborn, immature, and adult rabbit, 300

$\mathrm{Na}^{+} \mathrm{K}^{+}$-ATPase, renal activity, prolactin effect, neonatal rat, 207

Natural killer cell, decreased activity, very low birth weight infants, 376

Necrosis, intestinal, platelet activating factor role, rat, 428

Neonate

acid-base balance, varying degrees of acute hypoxia, swine, 112

$\beta$-adrenergic receptors, prolonged $\beta$-agonist infusion effect, lamb, 462

cardiomegaly, dexamethasone-induced, rat, 359 
cerebral flow velocity waveforms, perinatal assessment, 649

chronic hypoxemia, postnatal intestinal function during, lamb. 234

cytopenia, pathogenesis, during sepsis, 574 diaphragm, glycogen content, increased work load effect, lamb, 349

exogenous surfactant kinetics, after intratracheal instillation, piglet, 445

extracorporeal membrane oxygenation, effect on blood flow distribution, lamb, 80

full-term, plasma cholesterol and lathosterol levels, 396

glycerol metabolism, effect of maternal diabetes and intrauterine growth retardation, 52

glycogen content in diaphragmatic fibers, increased work load effect, neonatal lamb, 354

hemoglobinopathy screening, DNA confirmation, dried blood specimens, 217

hypercarbia, indomethacin effect on choroidal and retinal blood flow during, piglet, 127

leukotriene $\mathrm{D}_{4}$ role, GBS-induced pulmonary hypertension, lamb, 121

monocyte, defective production of interleukin- 6 by, 18

pulmonary circulation, pulsatile perfusion effect, lamb, 349

seizure, pancuronium bromide pretreatment effect, pig, 636

sick, thrombin regulation, 391

swallowing and breathing coordination, during suckle feeding, 619

$\mathrm{T}$ cells, decreased granulocyte-macrophage colony-stimulating factor production by, 211

triglyceride-fatty acid cycling, effect of maternal diabetes and intrauterine growth retardation, 52

Nephron, $\mathrm{Na}^{+} \mathrm{K}^{+}$-ATPase activity, prolactin effect, neonatal rat, 207

Nutritional status, maternal, fetal placental lactogen responses to changes in, sheep, 520

Ontogeny, erythropoiesis during, 170

Osteocalcin, bone mineral content prediction and, very low birth weight infants, 181

Oxygen

arterial saturation, fetal monitoring, reflectance pulse oximetry, lamb, 266

arterial tension, effect of varying, neonatal swine, 112

transcutaneous, premature and normal term infants, 73

Oxygen free radical, role, pathogenesis of pial arteriolar dysfunction, rat, 640

Pancreas, $\beta$-cells, regulation of mammalian glucose transporters,

Pancuronium bromide, pretreatment, seizure-induced cerebral hyperemia, newborn pig, 636

Pantothenate, role, prophylaxis of valproate hepatotoxicity, mouse, 419

Pearson's marrow-pancreas syndrome, blood mitochondrial DNA deletions, 557

Pentoxifylline

effect on acute pulmonary hypertension, piglet, 163

effect on cardiovascular manifestations, group B streptococcal sepsis, piglet, 596

-indomethacin pretreatment, group B streptococcal sepsis, piglet, 222
Peptide, gastrointestinal, response, during fasting and feeding, preterm infant, 587

Perfusion, pulsatile, pulmonary vascular resistance and, newborn lamb, 349

Phosphodiesterase, inhibitors, inotropic responses, rabbit myocardium, 300

Placenta, regulation of mammalian glucose transporters,

Placental lactogen, circulating, nutritional regulation, fetal sheep, 520

Platelet activating factor, role, endotoxin and hypoxia-induced intestinal necrosis, rat, 428

Platelet-derived growth factor, localization, during fetal lung development, rat, 323

Prematurity: see also Infant, preterm

airway structure-function, lamb, 157

lung mechanics, time dependence of, lamb, 276

surfactant therapy with ventilation, surfactant distribution and lung compliance after, rabbit lung, 270

Prostaglandin

CAMP accumulation and, rat perinatal lung fibroblast, 344

effect on mucus gel layer thickness, developing rat, 193

Prostaglandin $H$ synthase, effect on GBS, piglet lung and liver, 14

Protein

hepatic, synthesis, developmental changes and fasting effect, suckling rat, 247

whole-body parameters, tracer comparison, premature infants, 95

Proximal convoluted tubule, acidification, developmental changes, rabbit, 411

Pulse oximetry, reflectance, fetal lamb, 266

Respiration

increased work load, glycogen response, neonatal diaphragm, lamb, 354

rhythmic swallowing and, during suckle feeding, term neonate, 619

training, preterm infants, 613

Respiratory syncytial virus, effect on reflex apnea, young lamb, 381

Reye's syndrome, animal model, ibuprofen interaction with influenza infection and hyperammonemia, ferret, 258

Riboflavin, oral supplementation, medium chain acyl-CoA dehydrogenase deficiency, 39

Salmonella abortus equi, febrile response, carotid denervation effect, lamb, 107

Sarcoplasmic reticulum, calcium pump, developmental regulation, rabbit heart, 474

Seizure, cerebral blood flow changes during, pancuronium bromide pretreatment effect, newborn pig, 636

Sepsis

cardiovascular manifestations, pentoxifylline effect, piglet. 596

group B streptococcus, combined pentoxifylline and indomethacin pretreatment, piglet, 222

neonatal cytopenias during, pathogenesis of, 574

Short stature, cartilage-hair hypoplasia, evaluation and growth charts for, 176

SIDS: see Sudden infant death syndrome

Skeletal alkaline phosphatase, bone mineral content prediction and, very low birth weight infants, 18
Small intestine

postnatal function, alterations, chronic hypoxemia, newborn lamb, 234

regulation of mammalian glucose transporters, 1

Sodium, ion transport, fetal distal lung epithelium, rat, 318

Sodium-potassium, erythrocyte transport, cystic fibrosis, 425

Steroidogenesis, metalloporphyrins and, rat adrenal, 196

Streptococcus

group B

capsule role, pulmonary hypertension, piglet, 386

cardiovascular manifestations, pentoxifylline effect, piglet, 596

cerebral arteriolar response, oxygen free radical role, rat, 640

leukotriene $\mathrm{D}_{4}$ role, newborn lamb, 121

prostaglandin $\mathrm{H}$ synthase effect, piglet lung and liver, 14

sepsis, combined pentoxifylline and indomethacin pretreatment, piglet, 222

Stress, perinatal adaptation, capillary vs arterial plasma catecholamines, 579

Stroke volume, heart rate fluctuations and, preterm infants, 117

Suckle feeding, rhythmic swallowing and breathing during, term neonates, 619

Sudden infant death syndrome

cardiac $R-R$ intervals, dynamic analysis, 606

medium-chain acyl-CoA dehydrogenase deficiency and, G-985 mutation, 305

mucosal immune response, trachea and duodenum, 372

Surfactant

distribution, after surfactant therapy and ventilation, preterm rabbit lung, 270

exogenous, short-term distribution kinetics, neonatal piglet, 445

lavage, meconium aspiration syndrome, piglet, 625

protein A, blood group A epitope contained in, 364

therapy, ventilation with, preterm rabbit lung, 270

$T$ cells, decreased granulocyte-macrophage colony-stimulating factor production, human neonate, 211

The American Pediatric Society and The Society for Pediatric Research, annual meeting abstracts, $3 \mathrm{~A}$

Thrombin, inhibition, impairment, sick neonates, 391

Thyroid hormone, myocardial adrenergic $\beta$ receptor responsiveness, late gestation lamb, 468

Thyroid hormone receptor. RNA ontogeny and, thyroxine treatment effect, rat kidney, 330

Tissue, iron status, effect of in vtero insulin exposure, fetal rat, 64

Tissue factor apoprotein, expression, blood monocytes, during infection, infant, 567

Tracer, comparison, whole-body protein parameters, premature infants, 95

Trachea, immunoglobulin $M$ response, SIDS victims, 372

Transforming growth factor- $\alpha$

gene expression, late-gestation fetal rat lung, 286

RNA ontogeny and, thyroxine treatment effect, rat kidney, 330

Triacylglycerol, highly unsaturated, accumulation, early postnatal rat, 47 
Triglyceride-fatty acid, neonatal cycling, effect of maternal diabetes and intrauterine growth retardation, 52

3,5,3'-Triiodothyronine, source of $T_{3}$ sulfate, fetal rat tissues, 541

3,5,3'-Triiodothyronine sulfate, metabolism, fetal rat tissues, 541

Tropoelastin, gene expression, pulmonary vasculature, rat, 280
Tumor necrosis factor- $\alpha$, early human milk, 29

Tyrosinemia, type I, immunoblot analyses, 43

Uridyltransferase, regional activity, rat brain, 512

Valproate, hepatotoxicity, pantothenate and carnitine prophylaxis, mouse, 419
Vitamin D, absorption, chronic cholestasis, 146

Vitamin E, water-solubilized form, role, vitamin D absorption, chronic cholestasis, 146

Water, loss, ontogeny of epidermal barrier to, perinatal rat, 308

Wilson's disease, animal model, Long-Evans Cinnamon rat, 253 